\title{
Retorno em escolaridade no Paraná
}

Juliana Kikuchi Van Zaist* Luciano Nakabashi*

\section{Introdução}

A educação, um dos principais componentes que determinam a quantidade de capital humano em uma economia, é objeto de análise e discussão, principalmente pela sua importância no desenvolvimento de uma nação. Um bom exemplo é o Brasil, onde comumente reporta-se ao desempenho do sistema educacional como sendo um dos fatores responsáveis pelo baixo grau do seu desenvolvimento.

$\mathrm{Na}$ literatura, a educação brasileira é descrita como sendo de fraco desempenho e de considerável atraso se comparada a um país de padrão de desenvolvimento semelhante. Algumas características apresentadas pela população brasileira são suas reduzidas capacidades de realização de tarefas mais complexas e de tomada de decisões que necessitam de uma boa capacidade analítica. Ambas acabam limitando o crescimento econômico.

Diante disto, uma das apostas possíveis para reverter tal cenário é a melhora na qualidade de ensino. No entanto, ainda há dúvidas quanto a prioridades e alocação dos recursos, ou seja, como os recursos são escassos, deve-se priorizar o investimento educacional nos níveis que tenham maior potencial de alavancar o crescimento econômico. Sendo assim, o principal objetivo desse estudo é averiguar, no caso da economia paranaense, qual nível de ensino (fundamental, médio ou superior) é mais importante na geração de renda. Para tanto, será feita uma análise acerca dos retornos da educação de acordo com os diferentes níveis de ensino.

\section{Análise empírica do impacto da escolaridade no Paraná}

\subsection{Metodologia}

Tendo em vista que a análise dos retornos da educação gera a necessidade de ter como base de dados somente aqueles que possuem rendimento e, conseqüentemente, que estão trabalhando, isso traz a possibilidade de a amostra estar viesada.

A causa disto está na estratégia de demanda por emprego do agente, na qual, segundo HECKMAN (1974), o agente tem implícito um determinado salário de reserva que

\footnotetext{
* Economista pela UFPR.

** Doutor em economia e Professor do Departamento de Economia da UFPR. Endereço eletrônico: luciano.nakabashi@,ufpr.br
} 
serve de parâmetro para o indivíduo aceitar ou não participar do mercado de trabalho (a participação se dará caso o salário ofertado seja superior a este salário de reserva).

Assim, conforme apontado por SACHSIDA, LOUREIRO e MENDONÇA (2004), a não consideração desse fenômeno faz com que as estimativas por MQO sejam tendenciosas devido ao aparecimento do denominado viés de seleção amostral, uma vez que se é analisado de forma idêntica grupos que usam regras diferentes.

Um exemplo clássico disto é o objeto de estudo de HECKMAN (1974), que se refere ao rendimento de um grupo de mulheres, as quais escolhem se vão ou não trabalhar, sendo que a opção por trabalhar faz delas participantes da amostra.

Se as mulheres fizessem essa escolha de forma aleatória, seria possível usar MQO para estimar algum modelo para salários. No entanto, essa suposta aleatoriedade é pouco provável, pois as mulheres a quem fossem oferecidos baixos salários, provavelmente optariam por não trabalhar, fazendo com que o salário observado seja viesado para cima.

Uma solução para esse problema, que já é algo padrão para esse tipo de amostra, pode ser encontrada se existirem variáveis que afetam significativamente as chances de observação (chance de estar trabalhando), mas não afetam o salário oferecido como, por exemplo, o número de filhos que a mulher possui.

Desse modo, ao se usar uma equação de seleção que leva em consideração as variáveis que afetam a decisão de uma mulher entre trabalhar ou não é possível corrigir aquele "viés para cima". Isto é exatamente o que foi proposto por HECKMAN (1979).

\subsection{Base de dados}

As estimações, por MQO e por HECKMAN (1979), foram obtidas a partir dos microdados da Pesquisa Nacional por Amostra de Domicílio (PNAD) para o Paraná, referentes ao ano de 2005.

A seleção da amostra se deu com a utilização dos mesmos filtros utilizados por SACHSIDA, LOUREIRO e MENDONÇA (2004), a saber:

$\left.1^{\circ}\right)$ A amostra compreende somente homens entre 24 e 56 anos de idade, uma vez que suas decisões são menos complicadas com relação ao nível de escolaridade, por considerações de fertilidade;

$2^{\circ}$ ) São incluídas apenas pessoas que não estejam estudando ${ }^{7}$;

\footnotetext{
${ }^{7}$ Esse filtro é baseado no estudo de GRILICHES (1977).
} 
$3^{\circ}$ ) A observação que não possui uma ou mais informações sobre as variáveis independentes é descartada da $\operatorname{amostra}^{8}$;

$4^{\circ}$ ) Busca-se evitar que sejam incluídas na amostra pessoas que possuam um salário extremamente alto que possa viesar os resultados. Considera-se, portanto, apenas indivíduos que possuem um salário horário entre $\mathrm{R} \$ 1,00$ e $\mathrm{R} \$ 500,00$. Busca-se, também, evitar pessoas que não estejam trabalhando.

$5^{\circ}$ ) Não foram considerados os indivíduos que trabalham nos setores públicos e agrícolas devido à dinâmica própria regida por eles (SOARES e GONZAGA, 1999 apud SACHSIDA, LOUREIRO e MENDONÇA, 2004)

\section{Resultados econométricos}

Utilizou-se uma expansão da equação minceriana na estimação da equação de salários:

$$
\begin{aligned}
\log w & =\beta_{0}+\beta_{1} S+\beta_{2} \operatorname{Exp}+\beta_{3}(\operatorname{Exp})^{2}+\beta_{4} \operatorname{Raça}+\beta_{5}(S \times \text { Raça })+\beta_{6}(S \times \operatorname{Exp}) \\
& +\beta_{7}(\operatorname{Exp} \times \text { Raça })+\beta_{8}(S \times \text { Raça } \times \operatorname{Exp})+\beta_{11}(\text { Formal })+\beta_{12}(\text { Sind }) \\
& +\beta_{13}(\text { Csdo })+\varepsilon
\end{aligned}
$$

Onde:

Log $\mathrm{w}=\operatorname{logaritmo}$ do salário/hora mensal;

$\mathrm{S}=$ escolaridade

$\beta_{0}=$ constante;

$\operatorname{Exp}=$ anos de experiência do trabalhador';

Raça = representa uma variável dummy em que assume o valor 1 se o trabalhador é branco e caso contrário;

Formal = representa uma variável dummy em que assume o valor 1 se o trabalhador pertence ao setor formal e 0 caso contrário;

Sind $=$ Sindicato $=$ representa uma variável dummy em que assume o valor 1 se o indivíduo é associado a algum sindicato e 0 caso contrário;

Csdo $=$ Casado $=$ representa uma variável dummy em que assume o valor 1 se o indivíduo é casado e 0 caso contrário;

$\varepsilon \quad=$ erro da estimativa.

Entretanto, com a realização da regressão por nível, constatou-se que muitos dos coeficientes, tanto pelo método de Heckman quanto por MQO, apresentaram-se não significativos. Diante disto, verificou-se, através do Fator de Inflação da Variância no STATA

\footnotetext{
${ }^{8}$ Os autores utilizaram o mesmo procedimento de Heckman et alli (2000) e Soares e Gonzaga (1999).

${ }^{9}$ A experiência é calculada pela forma padrão: Exp $=$ Idade - escolaridade $-6 . \mathrm{O} \mathrm{n}^{\circ} 6$ representa a idade que a criança começa a estudar.
} 
9.1, a existência de multicolinearidade prejudicando os resultados estimados. Contudo, ao se retirar as interações (S x Raça; S x Exp; Exp x Raça; e S x Raça x Exp), o problema acaba por ser amenizado.

Assim, tendo em vista estes dois modelos (com as interações e sem as interações), utilizou-se o CIS (Critério de Informação de Schwarz) para selecionar o melhor deles. De acordo com os resultados, o modelo escolhido e apresentado na seqüência é o que não apresenta as interações ${ }^{10}$.

Com relação à equação de seleção, usou-se como variáveis explicativas a escolaridade do indivíduo ( $\underline{\mathrm{S}})$, a variável dummy Filhos em que assume o valor 1 se o trabalhador possui filhos e 0 caso contrário e a variável dummy Csdo em que assume o valor 1 se o indivíduo é casado e 0 caso contrário. A variável $\underline{\mathrm{SM}}$ (escolaridade da mãe), diferentemente de SACHSIDA, LOUREIRO e MENDONÇA, (2004), não foi utilizada nesse estudo, pois, como foi mencionado pelos próprios autores, "os estudos conhecidos feitos para o Brasil para explicar a participação na força de trabalho não levam em consideração essa variável”, o que dificulta a obtenção de conclusões a respeito. Outro motivo refere-se ao fato de que a consideração de tal variável acabaria por restringir ainda mais a amostra.

\subsection{Resultados obtidos por Heckman e MQO}

$\mathrm{Na}$ Tabela 1, a faixa S1 considera os indivíduos que possuem de 1 a 4 anos de estudo, S2 considera os que possuem de 5 a 8, S3 leva em conta aqueles que têm de 9 a 11 e S4 compreende os que possuem 12 anos ou mais de estudo. Assim, um indivíduo que possui de 1 a 4 anos de estudo, por exemplo, terá um retorno de 7,2\% por ano adicional de estudo (segundo o método de Heckman).

Então, ao analisar a tabela abaixo, no que se refere aos resultados obtidos por Heckman, verifica-se que um ano a mais de estudo gera, em média, um retorno de $7,2 \%$ na primeira faixa, de 7,5\% na segunda faixa, de $8,3 \%$ na terceira faixa e de $11,9 \%$ na última faixa. Isto indica que o nível que apresenta o maior retorno é o superior, entretanto, independentemente do nível do ensino, verifica-se que há um retorno elevado gerado pela educação.

\footnotetext{
$10 \mathrm{O}$ modelo de Heckman sem interação apresentou CIS $=5006.053$, enquanto que o modelo de Heckman com interação apresentou CIS $=5022.694$.
} 
Tabela 1. Equação de Salários por nível

\begin{tabular}{|c|c|c|}
\hline Variável & MQO & Heckman \\
\hline \multirow{2}{*}{ Constante } & 0.0569874 & -0.2365793 \\
\hline & $(0.0772222)$ & $(0.0755253)^{*}$ \\
\hline \multirow{2}{*}{ S1 } & 0.0554872 & 0.0720389 \\
\hline & $(0.0140472)^{*}$ & $(0.0127558)^{*}$ \\
\hline \multirow{2}{*}{ S2 } & 0.0624127 & 0.0752137 \\
\hline & $(0.0075899)^{*}$ & $(0.0071993)^{*}$ \\
\hline \multirow{2}{*}{ S3 } & 0.0727289 & 0.0832089 \\
\hline & $(0.0051644) *$ & $(0.0050749)^{*}$ \\
\hline \multirow{2}{*}{ S4 } & 0.1148049 & 0.1188561 \\
\hline & $(0.0041962) *$ & $(0.0043090)^{*}$ \\
\hline \multirow{2}{*}{ Exp } & 0.0257487 & 0.0205517 \\
\hline & $(0.0051004)^{*}$ & $(0.0048566)^{*}$ \\
\hline \multirow{2}{*}{$\operatorname{Exp}^{2}$} & -0.0003015 & -0.0002367 \\
\hline & $(0.0001011)^{*}$ & $(0.0000944)^{* *}$ \\
\hline \multirow{2}{*}{ Raça } & 0.0689661 & 0.0685231 \\
\hline & $(0.0259372)^{*}$ & $(0.0292028)^{* *}$ \\
\hline \multirow{2}{*}{ Formal } & 0.1205914 & 0.1705413 \\
\hline & $(0.0287706)^{*}$ & $(0.0265386)^{*}$ \\
\hline \multirow{2}{*}{ Sind } & 0.1620196 & 0.1672711 \\
\hline & $(0.0273903)^{*}$ & $(0.0259301)^{*}$ \\
\hline \multirow{2}{*}{ Csdo } & 0.1395139 & 0.2552689 \\
\hline & $(0.0280001)^{*}$ & $(0.0308882)^{*}$ \\
\hline $\mathrm{R}^{2}$ Ajustado & 0.4500 & \\
\hline Amostra & 1934 & 1934 \\
\hline CIS & & 5.006 .053 \\
\hline
\end{tabular}

FONTE: Elaboração Própria a partir dos microdados da PNAD 2005

NOTA: *Estatisticamente significativo ao nível de 1\%; **Estatisticamente significativo ao nível de $5 \%$; Valores entre parênteses correspondem ao desvio padrão.

O gráfico abaixo possibilita uma visão geral dos retornos por nível. Através dele juntamente com a Tabela 1, verifica-se que, da mesma forma que os retornos no estado do Paraná são maiores conforme aumenta o número de anos de estudo.

Gráfico 1. Retornos em escolaridade por nível (2005)

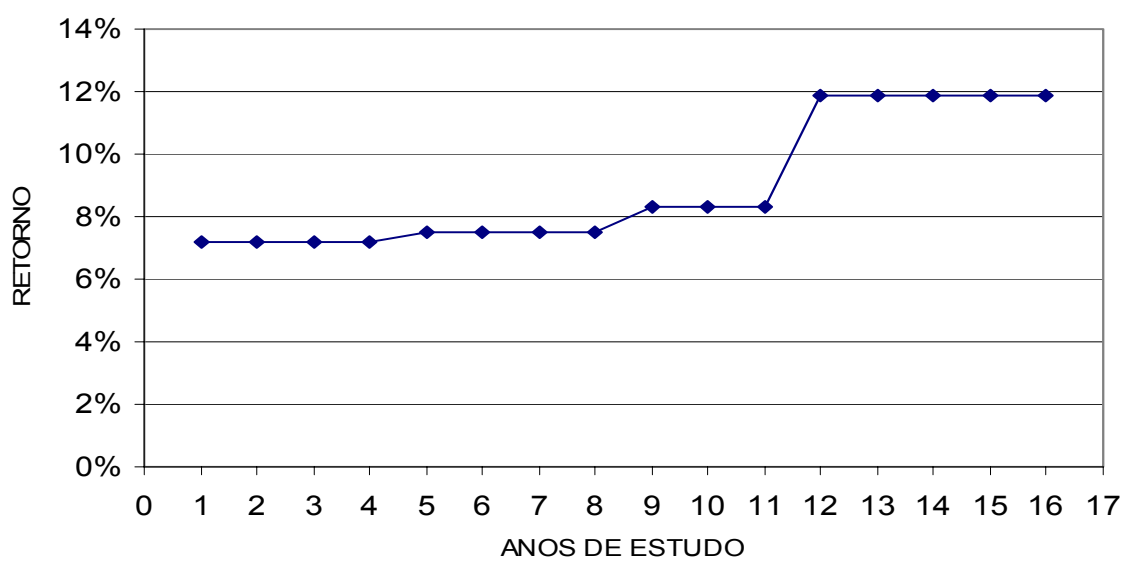

FONTE: Fonte: Elaboração própria a partir dos microdados da PNAD 2005. 
Com relação aos retornos obtidos por MQO, têm-se os seguintes resultados: 5,5\% para a primeira faixa, $6,2 \%$ para a segunda, $7,3 \%$ para a terceira e $11,5 \%$ para a última.

SACHSIDA, LOUREIRO e MENDONÇA (2004), a partir dos dados da PNAD de 1996, verificaram que o retorno pelo método de Heckman de 4 anos completos de estudo no Brasil é de 12,8\%, para 8 anos completos de estudo é de 14,2\%, para 12 anos completos é de 14,7\% e para 16 anos completos é de 14,8\%. Já por MQO foram de 16,6\%, 16,8\%, 17,0\% e $17,1 \%$, respectivamente.

BARBOSA FILHO e PESSÔA (2006) também realizaram um estudo do retorno da educação para o Brasil. Os dados por eles utilizados são da PNAD, do Censo Demográfico de 2000, da Pesquisa de Orçamento Familiar (POF) e do INEP. Os retornos identificados para um ano a mais de estudo em 2004, considerando 30 anos de experiência, foram os seguintes: 1) primário $=9,8 \% ; 2$ ) ensino fundamental (ou ginásio) $=14,8 \% ; 3$ ) ensino médio $=13,9 \%$; e 4) ensino superior $=13,8 \%$.

Para analisar qual o nível que traz mais benefícios os autores consideram, ainda, o prêmio salarial resultante de cada ciclo de educação que se completa, a saber: 1) primário = $11,9 \%$; 2 ) ensino fundamental $=14,9 \%$; 3 ) ensino médio $=16,4 \%$; e 4) ensino superior $=$ $32,8 \%$. Assim, verificaram que o nível em que se deve focar os investimentos é o superior ${ }^{11}$. Porém, por questões sociais, deixam claro que os dois primeiros níveis (primário e ginásio) não devem ser esquecidos.

Utilizando variáveis dummies, é possível verificar diferenças salariais para os diferentes níveis de estudo, tendo como base de comparação os indivíduos que não possuem o primário completo (indivíduos com menos de 4 anos de estudo), a saber:

\footnotetext{
11 Os autores também estudaram acerca do retorno da pré-escola que, da mesma forma que o superior, gera grandes benefícios e deve, portanto, também ser foco dos investimentos.
} 
Tabela 2. Diferenças Salariais

\begin{tabular}{lcc}
\hline \multicolumn{1}{c}{ VARIÁVEL } & MQO & HECKMAN \\
\hline Constante & 0.139033 & 0.2328417 \\
Primário & $(0.0729385)$ & $(0.0819898)^{*}$ \\
& 0.2116878 & 0.1993706 \\
Fundamental & $(0.0399902)^{*}$ & $(0.0401120)^{*}$ \\
& 0.4627066 & 0.4399585 \\
Médio & $(0.0470299)^{*}$ & $(0.0478048)^{*}$ \\
& 0.7464979 & 0.7163911 \\
Superior & $(0.0459288)^{*}$ & $(0.0474491)^{*}$ \\
& 1.725519 & 1.6837020 \\
Exp & $(0.0588701)^{*}$ & $(0.0612500)^{*}$ \\
& 0.0269667 & 0.0269292 \\
Exp & $(0.0052076)^{*}$ & $(0.0051956)^{*}$ \\
& -0.0003576 & -0.0003553 \\
Raça & $(0.0001033)^{*}$ & $(0.0001030)^{*}$ \\
& 0.086515 & 0.0854208 \\
Formal & $(0.0265047)^{*}$ & $(0.0267347)^{*}$ \\
& 0.123669 & 0.1226310 \\
Sind & $(0.0293691)^{*}$ & $(0.0292806)^{*}$ \\
& 0.1677507 & 0.1673769 \\
Csdo & $(0.0281044)^{*}$ & $(0.0280462)^{*}$ \\
& 0.1491153 & 0.1157359 \\
$\mathrm{R}^{2}$ Ajustado & $(0.0286925)^{*}$ & $(0.0317462)^{*}$ \\
\hline Amostra & 0.4226 & 1934 \\
\hline FONTE: Elaboração Própria a partir dos microdados da PNAD 2005 \\
NOTA: *Estatisticamente significativo ao nível de 1\%; \\
Valores entre parênteses correspondem ao desvio padrão.
\end{tabular}

Assim, pelo procedimento de Heckman, quem possui o primário completo $\left(1^{\mathrm{a}}\right.$ a $4^{\mathrm{a}}$ série) tem, em média, um salário 19,94\% maior do que quem não possui; quem possui o ensino fundamental completo ( $5^{\mathrm{a}}$ a $8^{\mathrm{a}}$ série) tem, em média, um salário 43,99\% maior do que quem não possui o primário; quem possui o ensino médio completo tem, em média, um salário $72 \%$ maior do que quem não possui o primário; e quem possui o superior completo tem um salário, em média, $168 \%$ maior do que quem não possui o primário ${ }^{12}$.

\section{Considerações Finais}

O presente trabalho buscou identificar qual nível de ensino é o mais importante na geração de renda no estado do Paraná através da estimação da equação de rendimentos com o

\footnotetext{
${ }^{12}$ Os resultados obtidos por MQO estão no apêndice deste trabalho.
} 
modelo de Heckman (1979), uma vez que estimativas por MQO não levam em conta decisões do agente quanto a participar ou não do mercado de trabalho, o que acaba por gerar o chamado viés de seleção amostral. Sendo assim, o modelo de MQO foi utilizado unicamente com o intuito de tê-lo como base de comparação.

Constatou-se que, assim como no Brasil, a educação no Paraná possui grande importância no rendimento do indivíduo. Os resultados mostraram que um ano adicional de estudo, por Heckman, gera um retorno de 7,2\% no primário ( $1^{\mathrm{a}}$ a $4^{\mathrm{a}}$ série), de 7,5\% no ensino fundamental ( $5^{\mathrm{a}}$ a $8^{\mathrm{a}}$ série), de $8,3 \%$ no ensino médio e de $11,9 \%$ no ensino superior. Por MQO, os retornos encontrados foram 5,5\%, 6,2\%, 7,3\% e 11,5\%, respectivamente.

Assim, detectou-se que, dentre os níveis de ensino, o superior foi o que demonstrou maior relevância (maior retorno). Isto indica que seria um erro, tanto por parte do governo, em seu papel de buscar melhoras no que se refere ao padrão de vida da população, quanto por parte do próprio indivíduo, em prol de seu benefício, deixar de investir neste nível.

No entanto, deixar de lado o ensino de base também seria um erro, uma vez que o menor retorno observado tem conseqüências no que se refere ao estímulo ao estudo. Isto implica que pessoas de baixa renda tendem a continuar nesta faixa, já que há um custo elevado de se estar estudando até que se obtenha maiores retornos. Neste caso, o papel do governo torna-se de extrema importância, tanto na ampliação da oferta do ensino público e melhoria de sua qualidade, quanto no desenvolvimento de estratégias e/ou políticas sociais visando tornar a opção pelo estudo uma escolha atrativa.

\section{Referências Bibliográficas}

GRILICHES, Zvi. Estimating the returns to schooling: Some econometrics problems. Econometrica, 1977, vol. $45, \mathrm{n}^{\circ} 1$. p. $1-22$.

HECKMAN, James J. Shadow prices, market wages and labor supply. Econometrica, 1974, vol. 42, $\mathrm{n}^{\circ} 4$, p. 679-694.

HECKMAN, James J. Sample selection bias as a specification error. Econometrica, 1979, vol. 47, $\mathrm{n}^{\circ}$ 1, p. 153-161.

PESSÔA, Samuel. A; BARBOSA FILHO, Fernando H. Retorno da Educação no Brasil. Seminário de Política Econômica e Social da FGV, 2006.

SACHSIDA, Adolfo; LOUREIRO, Paulo Roberto A; MENDONÇA, Mário Jorge C. Um Estudo Sobre Retorno em Escolaridade no Brasil. Rio de Janeiro, 2004. 